\title{
Is Endoscopic Ultrasound-Fine Needle Aspiration for Ki67 Aspirational Enough?
}

\author{
Yakira David ${ }^{1}$ and Michelle Kang Kim² \\ ${ }^{1}$ Henry D. Janowitz Division of Gastroenterology, ${ }^{2}$ Samuel Bronfman Department of Medicine, Icahn School of Medicine at Mount Sinai, New \\ York, NY, USA
}

See "The Role of Endoscopic Ultrasound-Guided Ki67 in the Management of Non-Functioning Pancreatic Neuroendocrine Tumors" by YongYan Cui, Lauren G. Khanna, Anjali Saqi, et al., on page 213-220.

Pancreatic neuroendocrine tumors (PNETs) are relatively rare with an estimated incidence of 2.2 cases per 1,000,000 in the US, of which $60 \%-90 \%$ are classified as non-functional. ${ }^{1}$ PNETs have deleterious effects such as hormone secretion (functional), metastases, or mass effects. Surgical resection is recommended for non-metastatic primary tumors that produce hormones, compress adjacent structures, or are $>2 \mathrm{~cm}^{2-4}$ However, due to frequent abdominal imaging, there has been a corresponding increase in the detection of small asymptomatic PNETs.

Defining the optimum management strategy for these incidentally found non-functional PNETs is challenging due to their heterogeneous behavior. There is an elevated risk of metastasis and mortality if high-risk lesions are not resected. Conversely, while most small PNETs are amenable to enucleation/local resection, others may require invasive procedures such as a pancreaticoduodenectomy or distal pancreatectomy with splenectomy. The potential morbidity associated with these procedures must be carefully considered in the case of low-grade lesions which are likely to have an indolent course.

Received: February 13, 2020 Accepted: February 20, 2020

Correspondence: Yakira David

Henry D. Janowitz Division of Gastroenterology, Icahn School of Medicine at Mount Sinai, 1 Gustave Levy Place, New York, NY 10029, USA

Tel: +1-917-995-5408, Fax: +1-254-233-8873, E-mail: yakira.david@mountsinai. org

ORCID: https://orcid.org/0000-0001-7232-0757

cc This is an Open Access article distributed under the terms of the Creative Commons Attribution Non-Commercial License (http://creativecommons.org/ licenses/by-nc/3.0) which permits unrestricted non-commercial use, distribution, and reproduction in any medium, provided the original work is properly cited.
Therefore, appropriate pre-operative risk-stratification of these lesions is challenging. Ki67 as a measure of proliferation rate has been found to be a strong prognostic indicator ${ }^{5}$ and is recommended by major society guidelines in conjunction with the mitotic index to adequately grade PNET lesions. ${ }^{2-4}$ The North American Neuroendocrine Tumor Society recommends that although needle biopsy or surgical resection specimens are optimal, fine needle aspiration (FNA) samples can provide adequate information in most cases. ${ }^{3}$ However, till date, the concordance rates of samples obtained by endoscopic ultrasound (EUS)-FNA and subsequent resected surgical specimens have been variable, ranging from $61 \%-84 \%$ in different studies. ${ }^{6}$

In this single center retrospective study titled "The Role of Endoscopic Ultrasound-Guided Ki67 in the Management of Non-Functioning Pancreatic Neuroendocrine Tumors", Cui et al. $^{7}$ found a $73 \%$ concordance rate between EUS-FNA cytology and surgical pathology in 37 samples. Concordance was highest for high-grade and low-grade lesions with positive predictive values of $100 \%$ and $80 \%$, respectively. However, this study demonstrates the limitation of EUS-FNA for intermediate-grade lesions with a positive predictive value of only $37 \%$. The authors appropriately acknowledge this limitation and further analyze the discordant specimens to identify features which would aid in the risk stratification of these lesions. Reassuringly, a majority of the lesions that were graded as intermediate-grade in EUS-FNA were downgraded after evaluation of the surgical specimen. The lone specimen that was upgraded from intermediate to high and 5 specimens that 
were upgraded from low to intermediate were all $>25 \mathrm{~mm}$ and would have otherwise met the criteria for surgical resection. As such, despite lesions being under-graded in EUS-FNA, it is unlikely that they would have been inappropriately excluded from surgery. However 3 of the 4 intermediate lesions that were downgraded at the time of surgery were $\leq 20 \mathrm{~mm}$ in size and could have potentially been considered for surveillance.

The major contributing factors cited for the observed discordance are intra-tumoral heterogeneity (especially in large lesions) and low cut-off values for the minimum number of cells counted. It is challenging to eliminate sampling errors, and the optimal method for tissue acquisition or the use of histology vs. cytology for tissue analysis is not well-defined. For instance, in the only other study conducted specifically in non-functional PNETs, a $19 \mathrm{G}$ needle was used to obtain all the tissue samples that were formalin-fixed and paraffin-embedded before measuring Ki67. ${ }^{8}$ Using this method, concordance between the samples obtained by EUS and by surgical resection was $83.3 \%{ }^{8}$ Moreover, other studies have identified that fine needle biopsy may have a higher cell yield and concordance rate than FNA. ${ }^{9}$ Additionally, while there was no difference in the number of cases between discordant lesions that were upgraded and those that were downgraded, it would be useful to assess if there was a difference between all the discordant and the concordant lesions. Therefore, there is scope to conduct further investigation to identify the technical aspects of tissue acquisition that result in the highest concordance rates.

Another contributing factor for low concordance rate reported in this study was the low cell count obtained by EUSFNA. As reported in prior studies, high cell counts lead to high concordance rates. ${ }^{10}$ It would be interesting to evaluate any significant difference in the cell count between the concordant and discordant specimens in this study which could help define the minimum cell count required to reliably grade these lesions.

With regards to their secondary outcomes, the finding that measures of proliferation such as Ki67 and mitotic index were reliable prognostic predictors of post-resection recurrence and disease-related death concurs with the existing literature. ${ }^{11}$ However, positive lymph nodes at the time of resection were not associated with poor prognosis. The prognostic value of lymph nodes has been variable in studies and is regarded as controversial in some guidelines. ${ }^{4}$ Additionally, lymph node involvement may be undetected as they are not consistently sampled particularly in lesions that were removed by enucleation. Other elements included in the American Joint Committee on Cancer staging such as lymphovascular invasion may have been associated with poor prognosis but were not significant factors. Thus, Ki67 can be used to guide post-oper- ative surveillance, and further trials should be performed to determine which patients should be considered for adjuvant chemotherapy even in the absence of lymphovascular or lymph node involvement.

In conclusion, this study demonstrates that EUS-FNA provides good concordance of Ki67 with surgically resected specimens in both high and low-grade lesions. Further, it demonstrates that the concordance in intermediate-grade lesions is low, but appropriate triage of these lesions to surgery or surveillance can be facilitated by considering other parameters such as tumor size. In addition, Ki67 and mitotic index were demonstrated to be the most reliable prognostic parameters. For adequate risk stratification and prognosis of these lesions, further studies are needed to evaluate the optimal technique for obtaining these tissue specimens to reduce inter-institution variability and sample bias due to intra-tumor heterogeneity.

Conflicts of Interest

The authors have no financial conflicts of interest.

\section{Author Contributions}

Conceptualization: Yakira David, Michelle Kang Kim

Supervision: MKK

Writing-original draft: YD

Writing-review\&editing: YD, MKK

ORCID

Michelle Kang Kim: https://orcid.org/0000-0001-5285-8218

\section{REFERENCES}

1. Halfdanarson TR, Rabe KG, Rubin J, Petersen GM. Pancreatic neuroendocrine tumors (PNETs): incidence, prognosis and recent trend toward improved survival. Ann Oncol 2008;19:1727-1733.

2. Shah MH, Goldner WS, Halfdanarson TR, et al. NCCN guidelines insights: neuroendocrine and adrenal tumors, version 2.2018. J Natl Compr Canc Netw 2018;16:693-702.

3. Strosberg JR, Halfdanarson TR, Bellizzi AM, et al. The North American neuroendocrine tumor society consensus guidelines for surveillance and medical management of midgut neuroendocrine tumors. Pancreas 2017;46:707-714.

4. Falconi M, Eriksson B, Kaltsas G, et al. ENETS consensus guidelines update for the management of patients with functional pancreatic neuroendocrine tumors and non-functional pancreatic neuroendocrine tumors. Neuroendocrinology 2016;103:153-171.

5. Martin-Perez E, Capdevila J, Castellano D, et al. Prognostic factors and long-term outcome of pancreatic neuroendocrine neoplasms: Ki-67 index shows a greater impact on survival than disease stage. The large experience of the Spanish National Tumor Registry (RGETNE). Neuroendocrinology 2013;98:156-168.

6. Yang G, Ji M, Chen J, et al. Surgery management for sporadic small ( $\leq 2$ $\mathrm{cm}$ ), non-functioning pancreatic neuroendocrine tumors: a consensus statement by the Chinese Study Group for Neuroendocrine Tumors (CSNET). Int J Oncol 2017;50:567-574.

7. Cui Y, Khanna LG, Saqi A, et al. The role of endoscopic ultrasound-guided Ki67 in the management of non-functioning pancreatic 
neuroendocrine tumors. Clin Endosc 2020;53:213-220.

8. Larghi A, Capurso G, Carnuccio A, et al. Ki-67 grading of nonfunctioning pancreatic neuroendocrine tumors on histologic samples obtained by EUS-guided fine-needle tissue acquisition: a prospective study. Gastrointest Endosc 2012;76:570-577.

9. Leeds JS, Nayar MK, Bekkali NLH, et al. Endoscopic ultrasound-guided fine-needle biopsy is superior to fine-needle aspiration in assessing pan- creatic neuroendocrine tumors. Endosc Int Open 2019;7:E1281-E1287.

10. Hasegawa T, Yamao K, Hijioka S, et al. Evaluation of Ki-67 index in EUS-FNA specimens for the assessment of malignancy risk in pancreatic neuroendocrine tumors. Endoscopy 2014;46:32-38.

11. Panzuto F, Boninsegna L, Fazio N, et al. Metastatic and locally advanced pancreatic endocrine carcinomas: analysis of factors associated with disease progression. J Clin Oncol 2011;29:2372-2377. 